\title{
Wharton's Jelly or Bone Marrow Mesenchymal Stromal Cells Improve Cardiac Function Following Myocardial Infarction for More Than 32 Weeks in a Rat Model: A Preliminary Report
}

\author{
Yelica López ${ }^{\#, 1}$, Barbara Lutjemeier ${ }^{\#, 1}$, Kiran Seshareddy ${ }^{1}$, Elizabeth M. Trevino ${ }^{1}$, K. Sue \\ Hageman $^{1}$, Timothy I. Musch ${ }^{1}$, Michele Borgarelli ${ }^{\#, 3,5}$ and Mark L. Weiss*,1,2,4 \\ ${ }^{I}$ Department of Anatomy \& Physiology; ${ }^{2}$ Midwest Institute for Comparative Stem Cell Biology; ${ }^{3}$ Department of Clinical \\ Sciences, College of Veterinary Medicine, Kansas State University, Manhattan, KS 66506, USA
}

\begin{abstract}
The therapeutic effect of mesenchymal stromal cells (MSCs) following myocardial infarction (MI) is small. This may be due to differences in cellular sources and donor age, route of administration, in vitro cellular manipulations and the short time course of follow up in many animal studies. Here, we compared MSCs from two different sources (adult bone marrow or Wharton's jelly from umbilical cord) for their long-term therapeutic effect following MI in a rat model to evaluate the effect of donor age. MSCs (or control infusions) were given intravenously 24-48 hr after myocardial ischemia (MI) induced by coronary artery ligation. Cardiac function was assessed by ultrasound at time points starting from before MSC infusion through 68 weeks after MI. A significant improvement in ejection fraction was seen in animals that received MSCs in time points 25 to 31 wks after treatment ( $<0.01$ ). These results support previous work that show that MSCs can cause improvement in cardiac function and extend that work by showing that the beneficial effects are durable. To investigate MSCs' cardiac differentiation potential, Wharton's jelly MSCs were co-cultured with fetal or adult bone-derived marrow MSCs. When Wharton's jelly MSCs were co-cultured with fetal MSCs, and not with adult MSCs, myotube structures were observed in two-three days and spontaneous contractions (beating) cells were observed in fiveseven days. The beating structures formed a functional syncytium indicated by coordinated contractions (beating) of independent nodes. Taken together, these results suggest that MSCs given 24-48 hr after MI have a significant and durable beneficial effect more than 25 weeks after MI and that MSC treatment can home to damaged tissue and improve heart function after intravenous infusion 24-48 hrs after MI, and that WJCs may be a useful source for off-the-shelf cellular therapy for MI.
\end{abstract}

Keywords: Bone marrow, cardiomyocyte differentiation, cellular therapy, mesenchymal stromal cells, myocardial ischemia, preclinical testing, rat, echocardiography, regenerative medicine, umbilical cord, Wharton's jelly.

\section{INTRODUCTION}

Ischemic heart disease is the primary cause of death worldwide [1]. Approximately 7.9 M American adults have had a myocardial infarction (MI). MI is caused primarily by occlusion of the coronary vessels and results in cardiomyocyte death, scar formation, ventricular remodeling, and eventual heart failure. In general, the myocardium does not regenerate lost cardiomyocytes and pharmacological interventions cannot resurrect dead myocardium. Alternatively, rescuing/repairing damaged myocardium by cellular therapy offers the possibility of treating MI and preventing heart failure.

A number of experimental and clinical investigations indicate that cellular therapy provides functional improvements to the heart following MI (see [2-4] for review).

\footnotetext{
*Address correspondence to this author at the Department of Anatomy and Physiology, Kansas State University, Coles Hall, room 105, Manhattan, KS 66506, USA; Tel: 785-532-4520; Fax: 785-532-4557;

E-mail: weiss@ vet.ksu.edu

Present Address: Department of Small Animal Clinical Sciences, VirginiaMaryland Regional College of Veterinary Medicine, Blacksburg, VA, USA

"These authors contributed equally to this work.
}

Cellular therapy following MI using mesenchymal stromal cells (MSCs) derived from bone marrow (BM-MSCs) demonstrate long-term cardiac rescue/repair in completed clinical trials [4-8]. In contrast, randomized, placebo controlled testing has revealed that mononuclear cell or hematopoietic progenitors do not produce sustained improvement [9-13]. A meta-analysis concluded that autologous MSC transplantation produces a significant modest improvement in cardiac function with no additional risks for 3-6 months following MI [7]. While experimental and clinical work supports the notion of cellular therapy, the effect is small [14].

MSC have classically been derived from the bone marrow cavity following bone marrow aspiration. Recently, MSCs have been found throughout the body including in placenta [15-19], adipose tissue [20-23] and umbilical cord [24-30]. MSCs can differentiate towards mesodermal lineages, such as fat, cartilage, bone [31-33]; neuroectodermal $[34,35]$, endodermal $[36,37]$ and cardiac cells $[38,39]$. In addition, MSCs can contribute to skeletal muscle following cell fusion events [40-42]. While MSC differentiation to cardiomyocytes is possible, human MSCs have low efficiency for cardiomyogenic differentiation [43, 44]. The age 
of the MSC donor may impact this observation since MSCs derived from younger rats have enhanced potential for cardiac differentiation [45]. Therefore, we speculated that myocardiogenic and therapeutic potential of MSCs depends upon tissue source and upon donor age.

Here, we tested whether MSCs derived from the Wharton's jelly of the umbilical cord (called Wharton's jelly cells or WJCs, below) would have enhanced therapeutic efficacy compared with MSCs from adult bone marrow (aBM-MSC) for their ability to improve cardiac function following transplantation in the rat MI model. While WJCs did not provide better preservation of cardiac function following treatment than aBM-MSCs, their sourcing, expansion and banking capabilities together suggest that WJCs are superior to aBMMSCs as an off-the-shelf treatment for treating MI. In a simplified cardiomyocyte differentiation assay, we found that after co-culture of WJCs and fetal BM-MSCs (fBM-MSCs) and not after co-culture of WJCs with aBM-MSCs, WJCs form simple beating structures (indicating differentiation to cardiomyocytes) in 5-7 days and more complex beating structures in 7-10 days. Together this preliminary report supports the contention MSCs have a durable positive therapeutic effect in MI and that WJCs may offer certain advantages over aBM-MSCs as cellular therapy for MI.

\section{MATERIAL AND METHODS}

\section{Isolation and Expansion of Rat Wharton's jelly-derived MSCs (WJCs)}

The method to isolate WJCs has been described previously [46]. Briefly, timed-pregnant female rats were euthanized humanely at 19-21 days post-coitus (DPC) and the uterus was removed aseptically to a sterile $150 \mathrm{~mm}$ culture dish. Inside a biosafety cabinet (BSC), the uterus was opened and the umbilical cords were collected (litters varied from 812 pups). The umbilical cord was rinsed with Dulbecco's Phosphate Buffered Saline (DPBS, Invitrogen, cat \# 14190250) to remove as much blood as possible, and blotted. The cord was moved to a single well of a six well dish $\left(8.7 \mathrm{~cm}^{2}\right)$ and torn into many small pieces (1 $\mathrm{mm}$ or smaller) using Dumont \#5 forceps. The pieces were allowed to dry in the well for about 10 minutes before DMEM with $15 \%$ fetal bovine serum (FBS) culture medium was added slowly. Over the next week, cells were seen migrating out from the cord explants onto the culture dish. At passage 0 , the cells were grown to $85 \%$ confluency prior to passage; at the first passage, the cells from all cords were pooled. To pass, the cells were rinsed twice with DPBS followed by $0.05 \%$ Tryp$\sin /$ EDTA for 5 minutes at $37^{\circ} \mathrm{C}$. The trypsin was inactivated by adding 3 times volumes of growth medium then the cells were pelleted by $200 \mathrm{~g}$ centrifugation for $5 \mathrm{~min}$ at room temperature. WJCs were used at passage 5-8 for transplantation studies. WJCs were transplanted by intravenous infusion via the lateral tail vein or the dorsal vein of the penis (IV, below) following labeling with SP-DiI (Invitrogen, D7777) using the manufacturer's protocol.

\section{Fetal Bone Marrow-Derived MSCs (fBM-MSCs)}

The pups that were harvested for deriving Wharton's jelly cells (WJCs) were used as fetal bone marrow donors.
Primary isolation of fetal rat bone marrow was obtained by removing the tibia and femur and slicing the bone into small pieces. These pieces were incubated in a $50 \mathrm{ml}$ centrifuge tube containing $5 \mathrm{ml}$ of $0.25 \%$ trypsin. During incubation bone pieces were mixed up and down by using $1 \mathrm{ml}$ pipette tip. Before centrifugation, the trypsinized tissues were filtered by using a 100 micron filter. Cells were centrifuged at $200 \mathrm{~g}$ for 5 minutes, then red blood cells were lysed using 1 $\mathrm{ml}$ of RBC lysing buffer for 1 minute. The cell suspension was pelleted and the cells were washed in DPBS, counted and suspended in growth medium (DMEM with 15\% FBS). Growth medium was changed every other day. At the first passage, all cells were pooled together as was done for the WJCs.

\section{Adult Bone marrow-Derived MSCs (aBM-MSCs)}

The timed-pregnant females which were euthanized to isolate WJCs were used as the adult bone marrow donor. The fore and hind limbs were rinsed with $70 \%$ ethanol, skinned, and amputated. Amputated limbs were placed into $150 \mathrm{~mm}$ sterile culture dish and moved to the BSC. Inside the BSC, the long bones were cleaned of muscle and rinsed with $70 \%$ ethanol. The ends of the bones were removed with bone scissors and the marrow was rinsed from the bones using a $25 \mathrm{ga}$ needle attached to a $10 \mathrm{cc}$ syringe containing DMEM. The bones were rinsed from both ends with $2-5 \mathrm{ml}$ of medium. The medium was collected, filtered through a $100 \mu \mathrm{m}$ sieve and centrifuged. The cell pellet was resuspended in medium and counted. BM-MSCs were plated at a density of 1015,000 cells $/ \mathrm{cm}^{2}$ in growth medium (DMEM with $15 \%$ FBS). The next day, any cells which were not attached were removed by a medium change. At passage 0 , the cells were grown to 80-85\% confluency prior to passage. In contrast to the WJCs and fBM-MSCs where all the cells from a litter were pooled, aBM-MSCs were processed and handled separately. To pass, the cells were rinsed twice with DPBS followed by $0.05 \%$ Trypsin/EDTA for 5 minutes at $37^{\circ} \mathrm{C}$. The trypsin was inactivated by adding 3 volumes of growth medium and the cells were pelleted by centrifugation. Adult BM-MSCs were used at passage 5-8. Adult BM-MSCs were transplanted intravenously via the lateral tail vein or the dorsal vein of the penis (IV, below) following labeling with SPDil (Invitrogen, D7777) using the manufacturer's protocol.

\section{Co-Culture WJC and BM-MSCs}

From 7 timed-pregnant rats, isolation of rat umbilical cords and fetal bone marrow were obtained at 19-21 DPC. Two umbilical cords were dissected into $2-3 \mathrm{~mm}$ pieces and were placed in a single well $\left(9.6 \mathrm{~cm}^{2}\right)$, after attachment 500 $\mu \mathrm{l}$ of growth medium was added. In the meantime, the skin and muscle were removed from the amputated leg of the fetus. Fetal BM-MSC cells were processed as described above. WJCs were co-cultured either with adult BM-MSCs or with fetal BM-MSCs. BM-MSCs were counted and placed at a density of 15,000 cells per $\mathrm{cm}^{2}$ over the explants. Then $1.5 \mathrm{ml}$ of warmed growth medium was added to the well. The co-cultures were observed daily for 7-10 days. In two out of seven isolations, the co-cultures were trypsinized and passaged as described above. In one out of seven isolations, fBM-MSCs were labeled with SYTO orange (Molecu- 
lar Probes) following the manufacturer's recommendations prior to co-culture with WJCs.

\section{Myocardial Infarction}

Animal protocol was reviewed and approved by the Kansas State University Institutional Animal Care and Use Committee (KSU IACUC, approval \#2614). MI was created by coronary artery ligation using previously described methods [47]. Briefly, male Sprague Dawley rats 4-6 months of age were anesthetized with isoflurane and intubated. The thorax was opened at the fourth or fifth intercostal space and the heart was exteriorized. The left main coronary artery of the rat was ligated with 9-0 nylon and the heart was returned to the thorax. The thorax was closed and the animal was extubated. The animals were closely monitored for $24 \mathrm{hr}$ after myocardial infarction. Timed-pregnant rats: Animal protocol was reviewed and approved by KSU IACUC (approval \# $3147,2614)$. The estrous cycle of female rats was monitored by vaginal cytology. When females were in proestrus, they were housed overnight with proven stud males. In the morning, the presence of sperm plugs was taken as proof of successful mating and that day was designated day 0 (days post coitus, DPC 0). On DPC 19-21, females were euthanized humanely and the reproductive tract was removed to harvest the umbilical cords and fetal bone marrow as described above. In some cases, the bone marrow from the adult females was isolated from the tibia, femur and humerus as described above. MSC transplantation: Animals were anesthetized with $2-3 \%$ isoflurane in $100 \%$ oxygen and placed in right lateral recumbence and heart function was measured by ultrasound 24-48 hrs after MI surgery. Rats were randomly assigned to one of three treatment groups: WJCs, aBMMSCs or vehicle (control). The WJC and BM-MSC groups received 3-10 × $10^{6}$ cells in $500 \mu \mathrm{l}$ of phosphate buffered saline (PBS) IV. The vehicle group received $500 \mu \mathrm{l}$ of PBS IV. Measurement of cardiac function: At various times following MI (from $24 \mathrm{hrs}$ through 68 weeks), cardiac function was assessed by ultrasound. Transthoracic echocardiography was performed under isoflurane (2-3\% in $100 \%$ oxygen) anesthesia by a board-certified veterinary cardiologist (MB) who was blind to the treatment group (WJCs, BM-MSCs, vehicle control). Rats were placed in right lateral recumbence and right parasternal long and short axis views of the heart were obtained. A Vivid 7 Dimension Cardiovascular Ultrasound System BT '06 (GE Healthcare, Milwaukee, WI, USA) equipped with a $11 \mathrm{Mhz}$ sectorial array transducer was used for image acquisition. Echocardiographic data were collected simultaneously with single-lead ECG and 2 dimensional (2D) cineloops including a minimum of 7 consecutive heart beats were digitally stored. Only measurements obtained from sinus beats were considered for further analyses. From the short axis right parasternal view at the level of the papillary muscle the following 2D and M-mode variables were measured: left ventricular (LV) internal diameter in diastole (LVDd), LV internal diameter in systole (LVDs) from which the fractional shortening was calculated. Measurements are reported as the average of 3-5 heart cycles. Left atrial diameter (LA), and aortic root diameter (Ao) were measured from the right parasternal short axis view at the level of the aortic root. From these, the LA/Ao ratio was calculated. Two-dimensional images were also obtained from the right parasternal long-axis four-chamber view and the right parasternal long-axis five-chamber view. From these images color-Doppler interrogation of the mitral and aortic valve was performed and calculation of ejection fraction was obtained. Ejection fraction (EF) was calculated with the area-length method from the right parasternal fourchamber view. In most cases, a second-generation ultrasound contrast agent BR- $38^{\circledR}$ was used (see pilot study in supplemental information). Ultrasound contrast agents are a suspension of stabilized gaseous microbubbles in saline. The gas inside the bubbles is perfluorocarbon gas. The product for intravenous infusion was prepared before use by adding $5 \mathrm{ml}$ of saline to the sealed vial followed by manual agitation for 30 seconds. BR-38 was infused through a $25 \mathrm{G} \mathrm{IV} \mathrm{cathe-}$ ter placed in the dorsal vein of the penis. The contrast was administered IV as a bolus at the dosage of $0.003 \mathrm{~mL} / \mathrm{kg}$ body weight. In order to avoid hemodynamic effects, a minimal volume (less than $0.2 \mathrm{~mL}$ including flush) was injected in each animal. MSC Homing In three MI animals and one normal animal, the homing of MSCs to the site of pathology was determined. In these cases, $4-10 \times 10^{6}$ SP-DiI (Invitrogen, D7777) labeled WJCs were injected IV 24 hours after MI. Four days later, the animals were euthanized and transcardially perfused with heparinized PBS $(150 \mathrm{mM}$ sodium chloride, $50 \mathrm{mM}$ Sorenson's phosphate buffer, $\mathrm{pH}$ 7.4, $3 \mathrm{IU}$ heparin per $\mathrm{ml}$ ) for $5 \mathrm{~min}$ to rinse out the blood followed by freshly prepared $4 \%$ paraformaldehyde in phosphate buffer ( $50 \mathrm{mM}$ Sorenson's phosphate buffer, $\mathrm{pH}$ 7.4) for $30 \mathrm{~min}$ for fixation. The hearts were removed and cryopreserved in $20 \%$ sucrose until they equilibrated. Using the ligature from the coronary artery ligation and the ischemic area for reference, the heart was cut into three blocks: root, middle section, and apex. The middle section that contained the ischemic zone distal to the ligature was sectioned further using a Leitz 1720 cryostat (12 micrometer sections) and air dried. The sections were hydrated and the nuclei were counterstained with Hoerscht 24638 (a fluorescent DNA stain), followed by being cleared in glycerol and coverslipped; adjacent sections were counterstained with methylene blue or hematoxylin and eosin, and coverslipped with DPX mounting medium (Sigma-Aldrich). Sections were observed using a Nikon Eclipse microscope fitted with Normaski differential contrast and epifluorescence illumination. Images were captured using a Roper CoolSnap ES using Metamorph V 7 software. TIFF Images were loaded into Canvas V10 software for plate construction. Montages were created by overlaying adjacent micrographs using tissue landmarks for registration.

\section{Data Analysis}

General linear mixed models were fitted to each of the following response variables: "ejection fraction, \%", "left ventricular end diastolic diameter", "left ventricular end systolic diameter", "fractional shortening, \%", or "left atrial diameter - aortic root diameter ratio" measured in rats at various times after induction of myocardial infarction. The linear predictor in the statistical model used for analysis included the fixed effect of cell therapy treatment (control, BM-MSCs, WJCs) and "time (in weeks) since induction of myocardial infarction", as well as their interaction. When evaluating ejection fraction, fractional shortening, left ven- 
tricular end diastolic diameter, left ventricular end systolic diameter, "Time" was modeled as a continuous variable to accommodate differing times of measurement amongst rats, ranging from 0 to 68 weeks after myocardial infarction. When evaluating left atrial diameter- aortic root diameter ratio, "Time was categorized into three intervals: Time (0-4 wk), Time (4-8 wks), Time (> 8wks). Also included in the model was a random blocking effect of group or litter, and its interaction with treatment to recognize the appropriate experimental unit for treatment. Kenward Roger's procedure was used to estimate degrees of freedom and adjust estimates for standard errors.

All statistical models were fitted using the MIXED procedure of SAS (Version 9.2, SAS Institute, Cary, NC). Model assumptions were evaluated using externally studentized residuals and were considered to be appropriately met. Following significant overall ANOVA, pairwise comparisons were conducted using post hoc tests. Note: When modeling "ejection fraction" and "fractional shortening", one observation was excluded from analyses based on a significant externally studentized residual, as per Bonferroni testing and an identification of influence on the fitted regression function. Based upon our previous experience with the myocardial infarction model and the small size of this preliminary study, significance was set at $p<0.10$ for main effects and interactions. Data are presented as means \pm one standard error of the mean (Figs. 3 and $\mathbf{6}$ ), or least square mean estimates (Fig. 5).

\section{RESULTS}

\section{MSC Characterization}

WJCs and adult or fetal BM-MSCs were characterized using three criteria provided by the International Society of Cellular Therapy (ISCT) [48]. First, MSCs were evaluated by morphology and self-renewal. WJCs and fetal and adult BM-MSCs (fBM-MSCs and aBM-MSCs) display typical MSC morphology and self-renewal (see Fig. 1). It was noted that aBM-MSCs had reduced self-renewal than the other groups as indicated by shorter lifespan in culture and more limited expansion potential then fBM-MSCs and WJCs. In addition, we noted more apoptotic cells and senescent cells in aBM-MSCs cultures. Examples of these observations are shown in Fig. (1) (compare Fig. 1c with 1a and b). Second, fetal bone marrow derived MSCs (fBM-MSCs) displayed MSCs surface markers CD90 (60.4\% positive), CD73 (68.2\% positive), and CD44 (23.9\% positive) and were negative for hematopoietic surface markers CD34 and CD45 (Fig. 1d). WJC expressed MSC surface markers CD90 (88.6\% positive), CD73 (46.0\% positive), and CD44 (37.8\% positive) and were negative for hematopoietic surface markers CD34 (0.0-0.1\%) and CD45 (0.0\%) (Fig. 1e). Third, WJCs and aBM-MSCs differentiated into bone, cartilage and fat using standard differentiation protocols and nonquantitative assessment (Fig. 2a-c). Compared to aBMMSCs, WJCs appeared to be more organized collagen fibers following chondrogenic differentiation (see Fig. 2a), Both WJCs and aBM-MSCs displayed osteogenic differentiation (Fig. 2b) and adipogenic differentiation (Fig. 2c). In summary, both WJCs and BM-MSCs met the minimum definition of MSCs as provided by the ISCT [32].

\section{Cardiogenic Potential Seen After Co-Culture of Bone Marrow MSCs and Wharton's Jelly Cells}

Twenty-four hours after initiation of co-culture of fetal BM-MSCs (fBM-MSCs) and WJCs, cells with tubular or cylindrical morphology were observed growing out from umbilical cord explant (see arrow in Fig. 2d), at 48-72 hour these structures start to elongate and form myotube-like structures (see arrows in Fig. 2e). At this time, multiple nuclei were observed in a central location (see Fig. 2k and 2l). Spontaneous contractions (beating) were observed in individual cells between days 5 and 7 (see Fig. 2f and supplemental video 1 ). The beating structures continued to organize until a functional syncytium was observed by day 10-12 post-isolation. This was indicated by independent clusters of beating cells that demonstrate coordinated contraction (see supplemental video 2 and 3). When beating cells were passaged after 10-12 days in co-culture, some cells continued beating till passage 2 . However, after passage, the cells were unable to organize into a functional syncytium (we did not observe network of coordinated contraction) and instead cells continued to beat as individuals. The present culture conditions do not maintain beating cells beyond passage 2 . As mentioned above, the beating myotubes in passage 0 and 1 were found to be multinucleated following DAPI staining and the tubular structures stained positive for muscle markers such as MyoD (see Fig. 2j-l). Additionally, the multinucleated cells had weak positive staining for connexin 43 (data not shown). These results are representative from six experiments (biological replicates) made using cell derived from either Fischer 344 or Sprague Dawley rats. In one experiment, adult BM-MSCs (aBM-MSCs) and WJCs were co-cultured; myotubes and beating structures were not observed (see Fig. 2d-f). In one experiment, placenta-derived MSCs and WJCs were co-cultured; no beating cells were observed (data not shown). In one experiment, fBM-MSCs were labeled with SYTO orange prior to co-culture with WJCs; this experiment suggested that WJCs, and not fBMMSCs, differentiated to the contracting structures (see supplemental Fig. 1).

\section{Effect of Myocardial Infarction (MI) on Heart Function}

To evaluate the effect of MI, EF of 4 normal healthy rats and 14 rats $24-48 \mathrm{hr}$ after MI was evaluated. As shown in Fig. 3, EF was decreased from $82.4 \pm 1.7 \%$ in normal, healthy control animals to $61.9 \pm 2.9 \% 24-48$ hrs after MI $\left(\mathrm{t}_{(16)}=-3.6, \mathrm{p}<0.01\right)$.

\section{Homing of WJCs to Damage}

To evaluate whether WJCs could be found in the heart after IV delivery, $3 \times 10^{6}$ WJCs labeled with SP-DiI were infused IV 24-48 hr after MI. To determine specificity of WJC homing, a healthy normal animal was injected with the same number of labeled WJCs IV. Four days after IV injection, all animals were sacrificed and the heart was removed, blocked, fixed, and cryoprotected. Frozen sections were collected from the peri-infarction zone, or the same region of the left ventricle of the normal rat, mounted on slides and either cleared and coverslipped for epifluorescent observation. As shown in Fig. (4), SP-DiI WJCs were found in the peri-infarction region in MI animals. In contrast, in normal, 

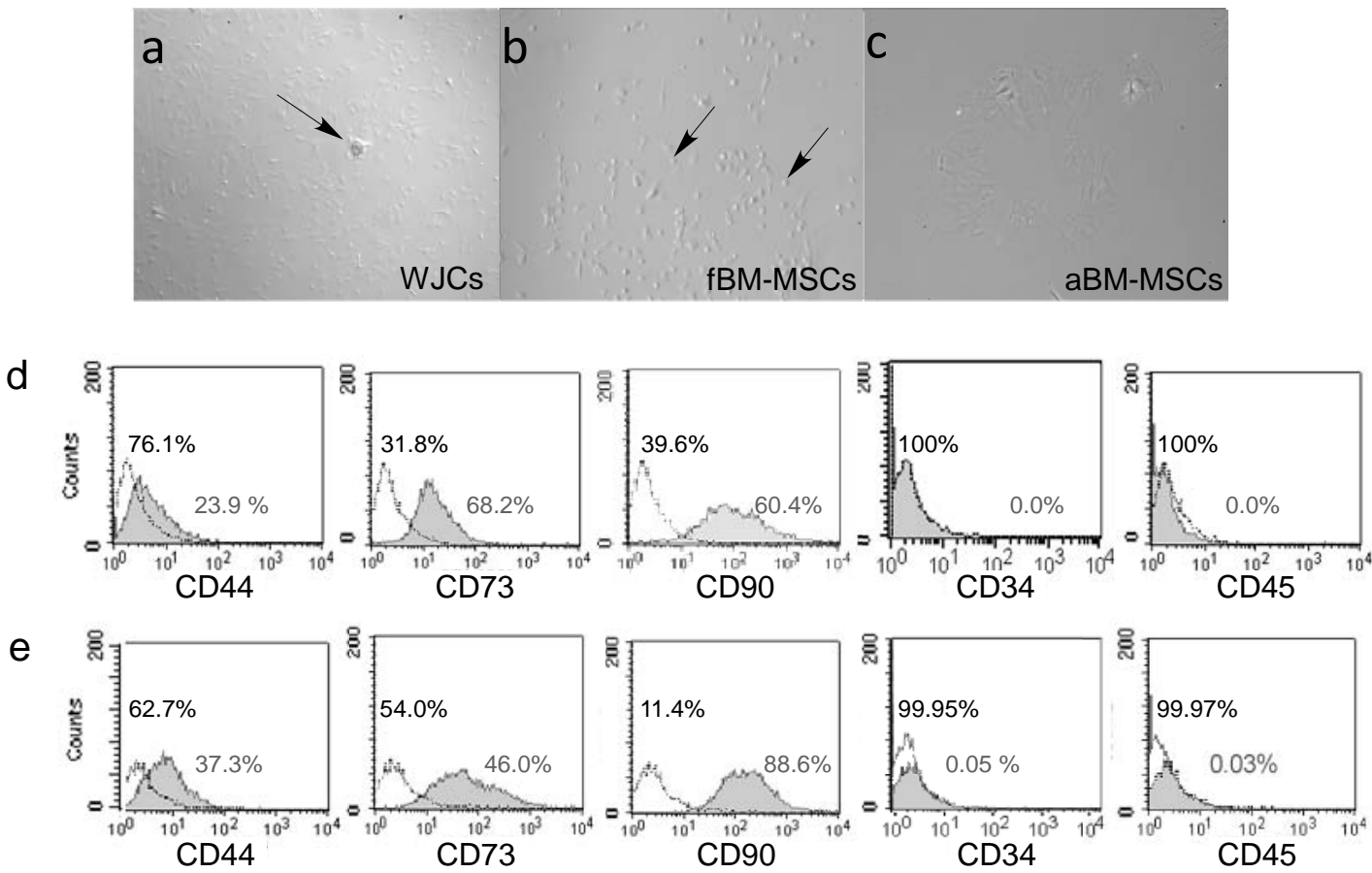

Fig. (1). Morphology and flow cytometry of mesenchymal stromal cells (MSCs). Primary isolation of rat Wharton's jelly derivedmesenchymal stromal cells (WJCs, a), fetal and adult bone marrow derived- mesenchmal stromal cells (fBM-MSCs and aBM-MSCs; b and c, respectively) derived from a Fischer 344 rat at passage zero. a) WJCs. WJCs show spindle-shape or stellate morphology typical of MSCs. Note: the arrow in a) indicates a colony forming unit-fibroblast (CFU-F). b) fBM-MSCs. fBM-MSCs show spindle-shape cells as well as round cells (arrows in b). c) aBM-MSCs. aBM-MSCs show flatted morphology, double nuclei, and apoptotic-related changes. aBM-MSC culture demonstrated lower expansion potential and early senescence (data not shown). All the pictures were taken 48 hour after isolation. (D and E) Flow cytometry of mesenchymal stromal cells derived from adult bone marrow (aBM-MSCs, d) or Wharton's jelly cells (WJCs, e). Flow cytometry was performed on passage 3 cells. Plot shows isotype control staining (open histogram) and specific antibody staining profile (gray histogram). Adult BM-MSCs and WJCs express CD44, CD73, CD90 and do not express CD34 or CD45.

healthy control rats that received SP-DiI WJCs IV, no SPDiI labeled cells were found in the heart (data not shown). In summary, WJCs were found around the damaged regions of the heart following IV injection and not in the normal healthy heart tissue in a control animal.

\section{Effect of MSCs on Cardiac Function Following Myocar- dial Infarction (MI)}

In this preliminary study, fourteen rats received MI and were randomly assigned to three experimental groups: four animals were controls and received phosphate buffered saline (PBS) infusion IV, four received WJCs (dose of $10 \times 10^{6}$ cells/ rat), and six rats received aBM-MSCs (dose of $10 \mathrm{x}$ $10^{6}$ cells/ rat). The IV infusions were given $24-48 \mathrm{hrs}$ after $\mathrm{MI}$ and after echocardiography. At various times $(2-68 \mathrm{wks}$ after infusion), heart function was assessed as described in Materials and Methods (see also the supplemental information). For each measurement, a 1-4 point qualitative value was assigned for the quality of the measurement, and each measurement was made twice both with and without contrast medium. To analyze the data, we used the values with the highest quality value, or in the case of a tie, the default was contrast-enhanced measurements. This resulted in using contrast-enhanced echocardiographic measures in all but two measurements.

\section{Ejection Fraction (EF)}

A generalized linear mixed model revealed that main effects Treatment, Time and Treatment $\mathrm{x}$ time interaction term were significant. As shown in Fig. (5a), post hoc analysis revealed that there was no evidence for an effect of MSCs at times $<25 \mathrm{wk}$. In contrast, BM-MSC and WJCs significantly improved ejection fraction at time points $>25 \mathrm{wks}$ ( $\mathrm{p}$ $<0.01)$. The significant positive effect of MSCs on EF was durable (25 - $32 \mathrm{wk})$. Furthermore, the MSC treated animals survived 52-68 weeks and the controls did not survive longer than 32 weeks so we were unable to compare directly the cardiac function at those time points. Note that despite randomization of group assignment, post hoc analysis revealed that the animals which received MSCs had decreased EF compared to vehicle control group at time $0(57.8 \pm 3.1 \%$ vs $72.0 \pm 3.4 \%, \mathrm{p}<0.05)$, see Fig. (6).

\section{Left Atria/ Aortic Root Diameter Ratio (LA/Ao)}

A generalized linear mixed model revealed that the main effects Treatment and Time were significant. As shown in Fig. (5b), animals that received WJCs showed decreased LA/Ao ratio relative to vehicle controls $\left(\mathrm{t}_{(13.5)}=2.24, \mathrm{p}<\right.$ 0.09 ) at all times. In contrast, BM-MSCs were not significantly different from vehicle controls. LA/ Ao ratio increased over time after MI in all groups $(\mathrm{p}<0.01)$. Other variables In contrast to $\mathrm{EF}$ and LA/Ao ratio, no significant 

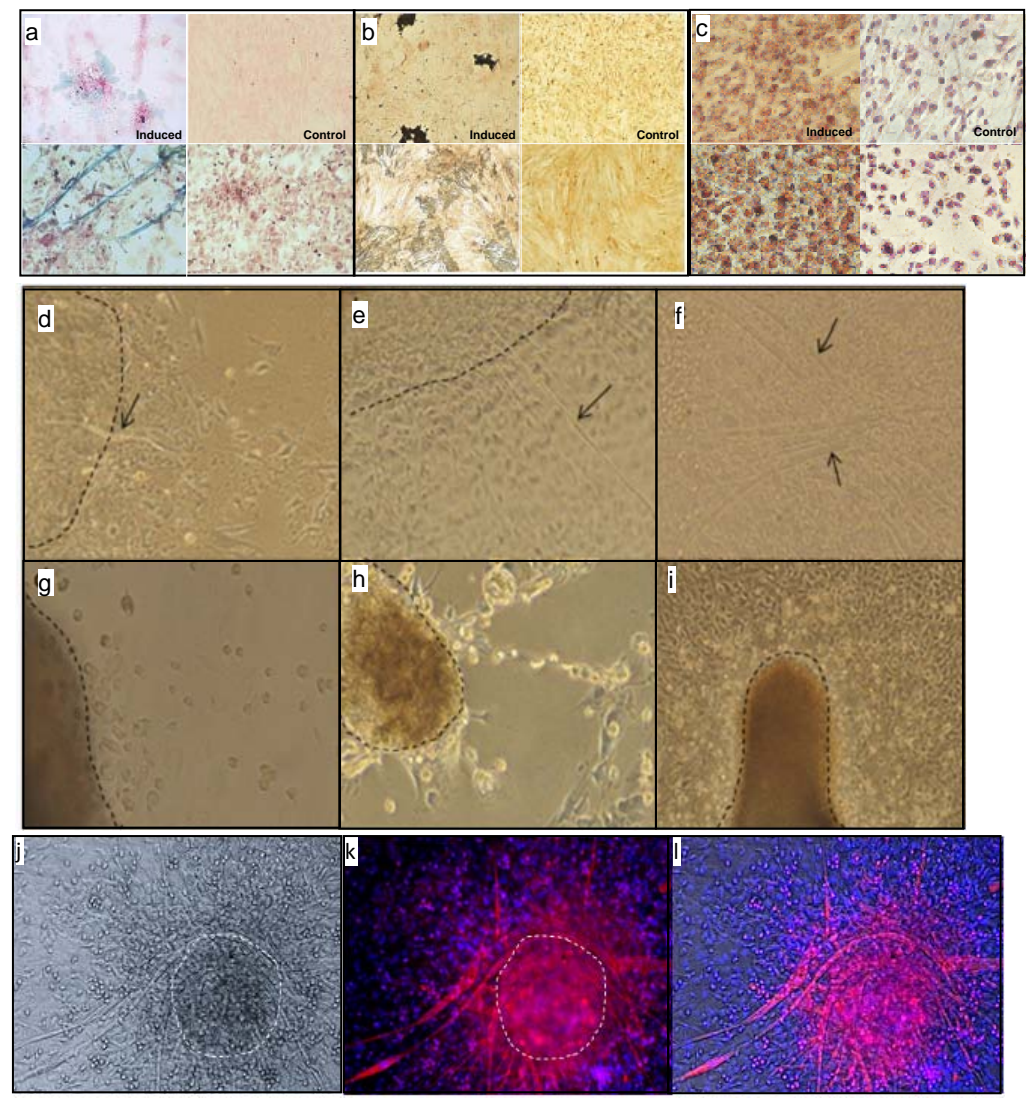

Fig. (2). a-c) Differentiation induced by incubation in chondrogenic differentiation medium (a), in osteogenic differentiation medium (b) or adipogenic differentiation medium (c). a)TOP: Adult bone marrow derived mesenchymal stromal cells (aBM-MSCs) maintained in chondrogenic differentiation medium (induced; left) or control medium (undifferentiated; right). a) BOTTOM: Wharton's jelly-derived MSCs (WCs) maintained in chondrogenic differentiation medium (induced, left) or control conditions (undifferentiated,right). Cells were differentiated for 21 days prior to being fixed and stained using Mason's Trichrome staining for collagen detection. Note that BM-MSCs showpositive staining (light green), which indicates the presence of collagen. Note that WJCs maintained in chondrogenic medium (left) displayed fascicules of fibrilar material staining positive for collagen. B) TOP: BM-MSCs maintained in osteogenic differentiation medium (left, induced) or control (undifferentiated) medium (right, control). b) BOTTOM: WJCs maintained in osteogenic differentiation medium (left) or control (undifferentiated) conditions (right). Cells were differentiated for 21 days prior to being fixed and von Kossa staining. Note the dark crystalline staining in the differentiated cells indicating deposition of calcium matrix. C) TOP: Adipogenic differentiation of BM-MSCs. Cells were cultured under adipogenic differentiation conditions (left, induced) or under standard culture (control) medium (right) for 21 days. C) BOTTOM: Adipogenic differentiation of WJCs. Adipogeneic conditions (induced) or standard culture conditions (control) for 21 days. Oil Red-O was used to stain lipids and hematoxylin for staining nuclei. Differentiation to adipogenic lineage induced small lipid droplets which stained positive with oil red-O to accumulate in the cytoplasm. d-i) Co-culture of rat Wharton's jelly cells (WJCs) with bone marrow derived mesenchymal stromal cells (BM-MSCs). Co-culture of WJCs with fetal BM-MSCs (fBM-MSC, d-f, j-1) or adult BM-MSCs (aBM-MSCs, g-i). Primary isolation and co-culture of WJCs with fBM-MSCs. d) Day 1 of co-culture. Note that a cylindrical structure (arrow in a) is observed growing from the umbilical cord explant (dashed line in a outlines umbilical cord explant). e) Day 4 of co-culture. Note that the cylindrical structure has enlarged and multiple nucleiare observed in the central portion. f) Day 7 of co-culture. Beating myotubes can be seen (beating structures are indicated by arrows). Note that multiple fibers are organized in parallel. g- i) Primary isolation and co-culture of WJCs and aBM-MSCs. g) Day 1 of co-culture. h) Day 4 of co-culture; and i) Day 7 of co-culture. Neither beating structures nor cylindrical structures were observed. j-1 Immunocytochemistry of WJCs co-cultured with fBM-MSCs. j) Phase contrast micrograph of WJCs with fBM-MSCs, dashed line indicates the boundary of rat umbilical cord explant. Note that multinucleated, myotube-like strucutures are observed growing from the explant. k) Immunocytochemical staining for MyoD (visualized using Alexa 594 secondary antibody) counterstained with 4',6diamidino-2-phenylindole (DAPI, a fluorescent DNA stain) revealed positive MyoD staining in both myotube-like structures, as well as in smaller fusiform or round uninucleated cells present in the center of the explant and periphery 1) Merge fluorescent markers with corresponding phase contrast to emphazise Myo D distribution associated with tube structures radiating from explant.

Treatment main effect or Treatment $\mathrm{x}$ time interaction effect was observed in Fractional Shortening (FS, $p=0.23$ ), left ventricular end systolic diameter (LVs, $p=0.61$ ), left ventricular end diastolic diameter $(\mathrm{LVd}, \mathrm{p}=0.74)(\mathrm{LVd}$ shown as representative in Fig. 5c). Consistent with our results for $\mathrm{EF}$ and LA/Ao, animals from all groups showed a significant change in the measurements over time following MI (FS, LVs and LVd, $\mathrm{p}<0.01)$. 


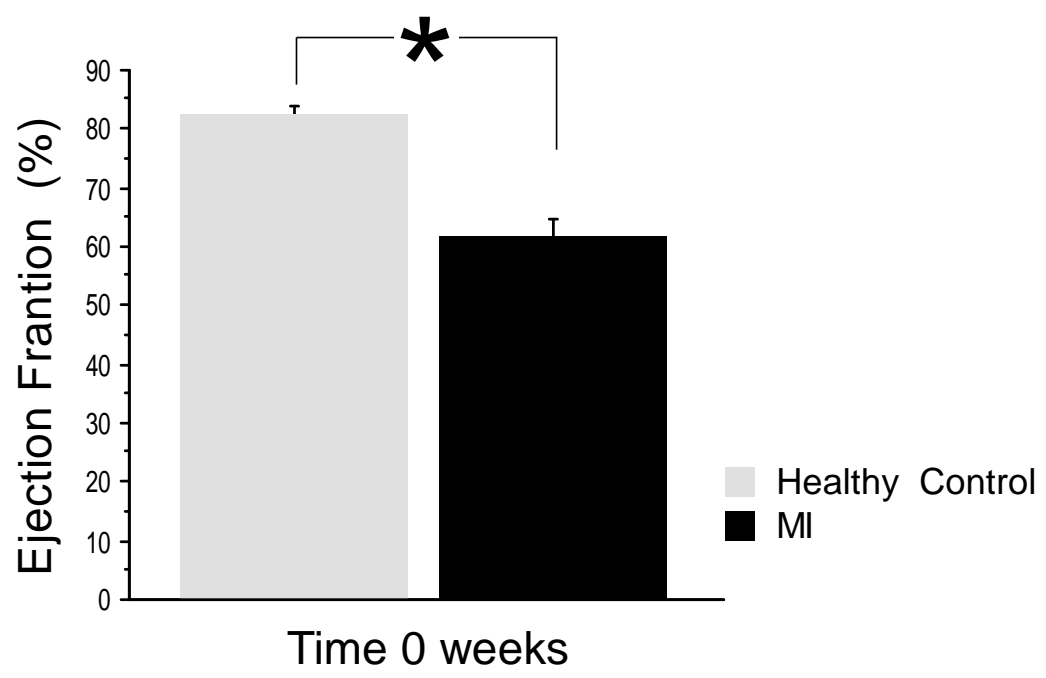

Fig. (3). Myocardial infarction (MI) decreases heart function. Twenty-four to 48 hours after MI, rats were lightly anesthetized for restraint and echocardiography was performed to measure heart function (ejection fraction). (* $\mathrm{p}<0.01)$.

We did not perform survival analysis due to the small number of animals in our preliminary study.

\section{DISCUSSION}

The major findings in the present investigation were that 1) WJCs home specifically to damaged myocardium after IV injection, 2) MSCs from adult bone marrow and from Wharton's jelly had a beneficial effect on the EF > 25 wk following MI and these effects are durable, extending from 25-32 wks after MI, and 3) 4) co-culture of WJCs with fetal BMMSCs, and not co-culture of WJCs with adult MSCs, induced the formation of myotube structures which contract rhythmically (beat) and formed a functional syncytium within one week.

To our knowledge, this is the first report of WJCs isolated from rats. Perhaps this is because the explant method is needed to isolate rat WJCs since enzymatic digestion of the cord is unreliable at establishing WJC cultures from rat umbilical cord [30]. In terms of morphology and self-renewal potential WJCs were more similar to fBM-MSCs than to aBM-MSCs (see Fig. 1). Both WJCs and BM-MSCs express CD73, CD90 and CD44. However, the surface expression of CD90 and CD44 reported here might be lower than for WJCs from human origin [30]. WJCs were multipotent in the qualitative assays performed in the present investigation; WJCs and BM-MSCs differentiate to bone, cartilage and fat. We suggest that there might be differences in the differentiation potential between WJCs and aBM-MSCs that more sensitive, quantitative assays might elucidate. In agreement with our differentiation findings, human WJCs produce more collagen than aBM-MSCs when placed under chondrogenic differentiation [33]. In contrast to our observation of rat WJCs differentiation to bone, human WJCs produce dense bone nodules, similar to aBM-MSCs [20]. Therefore, based upon their self-renewal and morphology, surface marker expression, and in vitro multipotency rat WJCs meet the definition of MSCs as defined by the ISCT [32].
There are a number of clinical trials evaluating MSCs as regenerative therapy following MI (90 clinical trials were found on the CLINICALTRIAL.GOV website using the search terms: "myocardial infarction" and "MSC" on 25 Sept 2012). To date, the experimental data suggests that MSCs from younger animals will provide greater benefit than those from older animals for treating MI [33-35]. A meta-analysis found that following intracoronary infusion of autologous BM-MSCs, there is a small, significant improvement in ejection fraction between 3 and 6 months with no enhanced risk [8]. Like intracoronary infusion of MSCs, IV infusion of BM-MSCs results in MSC homing to the heart, improves left ventricular function and decreases infarct size $[49,50]$. We found a significant improvement in ejection fraction at $25-$ 32 wks following IV infusion of aBM-MSCs or WJCs. Furthermore, the animals that received MSCs survived until till 68 weeks post-MI. In contrast, the vehicle control animals died $\leq 32$ weeks after MI. Similar to our observation using rat WJCs, Wu et al. tested human WJCs in a rat MI model and found positive effects on heart function in the 2-4 week survival period following direct transplantation into the heart [51]. At four weeks after transplantation, $\mathrm{Wu}$ et al. found engrafted human WJCs that stained with cardiac troponin T, suggesting that human WJCs show engraftment and in vivo cardiomyogenic potential [51]. Together with our data, these findings support the notion that MSCs given IV can possibly improve cardiac function following MI.

A number of mechanisms have been proposed for the improvement in cardiac function following MSC treatment (see review [52-55]). First, MSCs have a well-documented immune suppressive effect on activated $\mathrm{T}$ cell proliferation $[30,56-58]$. The thought is that immune modulation by MSCs would decrease immune-mediated cardiomyocyte losses following MI and reduce the size of the scar [59]. Second, MSCs have an angiogenic / neovascularization effect and generation of new vessels in the peri-infarct region may stem cardiomyocyte loss and aid in functional recovery following MI [51, 59-65]. Third, MSCs, especially MSCs 


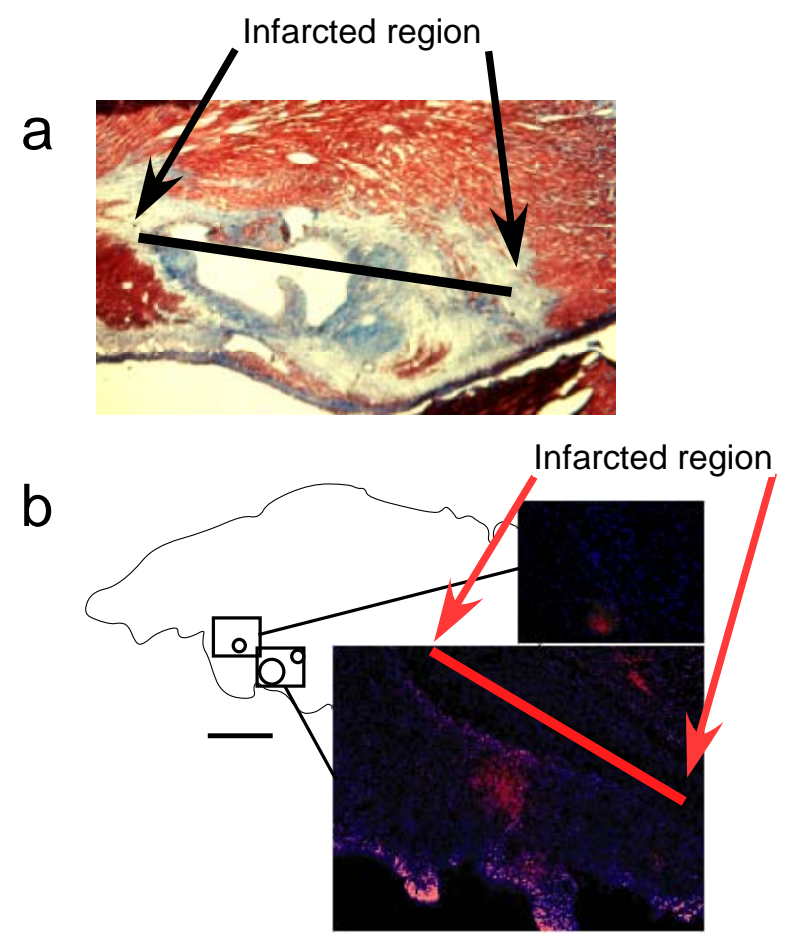

Fig. (4). Wharton's jelly cells (WJCs) home to the peri-infarct region following myocardial infarction (MI). Twenty-four hours after MI, rats received $3 \times 10^{6} \mathrm{Sp}$-DiI labeled WJCs by intravenous infusion through the dorsal vein of the penis. Four days later the animal was euthanized and the tissue was prepared as described in the Material and Methods. A. Tissue stained with hematoxylin and eosin showing the peri-infact region. B. Section adjacent to A stained with Hoerscht 24638 (a fluorescent DNA stain), cleared in xylenes and coverslipped for observation using epifluorescence. The heart outline is shown in the line drawing with box insets with circles the location of WJC clusters in the adjacent photomicrographs. Note that clusters of SP-DiI stained cells were located in the peri-infarct region. No SP-DiI labeled cells were found in the hearts after intravenous infusion of SP-DiI labeled WJCs in normal animals (not shown). Black bar $=1 \mathrm{~mm}$ scaling.

from a young donor, may have an enhanced cardiomyogenic potential in vitro $[38,44,45,66-71]$ and perhaps in vivo [51]. This mechanism suggests that donor cells form new cardiomyocytes to assist with heart function. Care must be taken in interpreting these data since MSCs might fuse with cardiomyocytes, and appear to be new cardiomyocytes [72, 73]. Fourth, MSCs produce a number of cytokines and chemokines which may reduce cardiomyocyte loss or rescue damaged cardiomyocytes from degeneration. This would suggest that MSCs might work at a distance to produce a beneficial effect following MI, and would not require MSCs to engraft in the heart to produce a positive effect. Note that MSCs "working at a distance" mechanism has been demonstrated following cornea damage; specifically the underlying mechanism was shown to be mediated by the antiinflammatory protein TNF- $\alpha$ stimulated gene / protein 6 released by MSCs [54]. Further work is needed to determine whether different types of MSCs have different functional benefits following MI, as has been suggested from work using smooth muscle cells to treat MI [65], and to determine which mechanism or mechanisms are responsible for our observations.

\section{Cardiomyogenic Potential of MSC}

The ISCT defines MSCs as cells that have a certain morphology and self-renew, express certain surface markers, and differentiate along mesoderm lineages such as bone, carti- lage and fat [32). MSCs, especially MSCs from young sources, have the potential to differentiate into neural lineages $[74,75]$ and myocardial lineage. Here, we demonstrated cardiomyogenic differentiation following co-culture of WJCs with fBM-MSC. Previous methods used to induce MSCs to cardiomyogenic differentiation include exposure to DNA methyltransferase inhibitor such as 5-azacytidine [44, $51,70,71,76,77]$, exposure to growth factors/ cytokines such as bone morphogenic protein 2 , fibroblast growth factor 2 , insulin-like growth factor $1[45,78]$, exposure to TGF- $\beta$ [79], or by co-culturing with cardiac myocytes [80-83]. In these methods, young MSCs, and to a lesser extent old MSCs differentiate into cardiomytocytes or precardiomyocytes over 2-3 wks or up to 30 days and these methods sometimes result in beating cells (such as shown in supplemental video 1). Infrequently did these methods produce coordinating beating as shown here in supplemental video 2 and 3 .

In contrast, in the present paper, our method induces rapid cardiogenic differentiation that may be more rapid than previous methods. By tracking migration of cells from the umbilical cord explant, we believe that the cardiogenic cells derive from WJCs (see supplemental Fig. 1), but more work is needed to support this conjecture. Our data indicates that the age of the donor impacts the MSC's ability to undergo cardiogenic differentiation since WJC and fBM-MSC coculture resulted in cardiomyogenic differentiation and syn- 
a

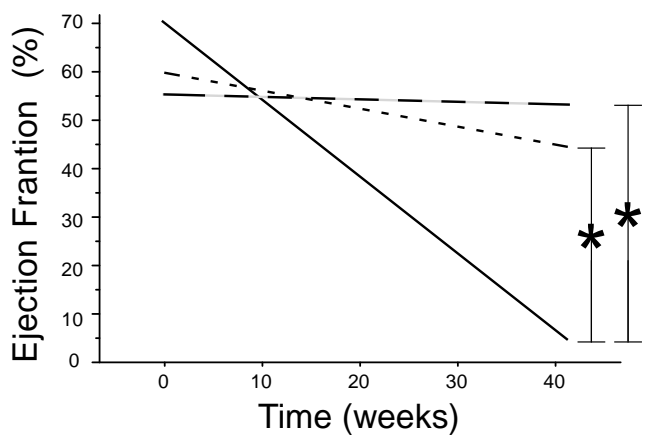

b

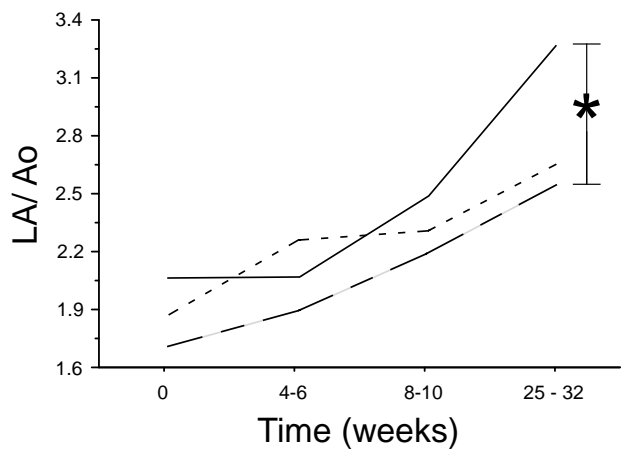

C

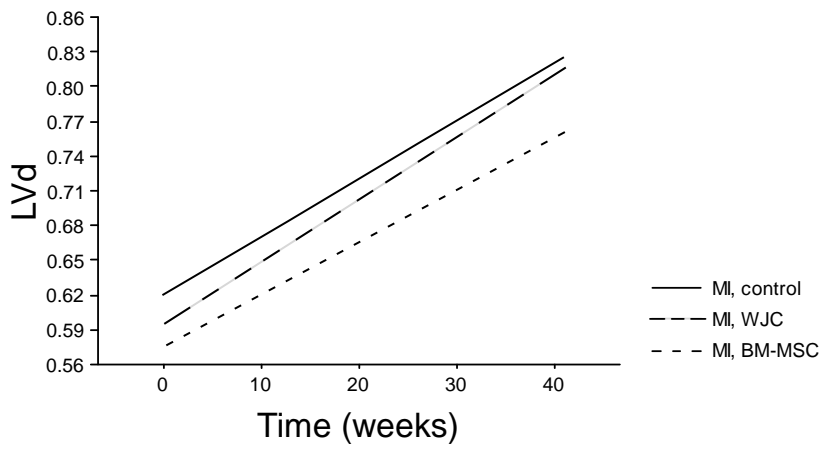

Fig. (5). Effect of mesenchymal stromal cell (MSC) treatment on heart function following myocardial infarction (MI). At various time periods (just before IV infusion of cells or control (time 0), and 2-32 weeks after MI), rats in all three experimental groups had echocardiographic assessment of cardiac function with and without contrast medium while under light anesthetic restraint. a) Ejection fraction. Ejection fraction in the control group decreased continuously until either death due to heart failure or termination at the end of the experiment. The decrease in ejection fraction in the two groups that received MSCs, either Wharton's jelly cells (WJCs) or adult bone marrow derived mesenchymal stromal cells (BM-MSCs), was significantly less than the control group at time points > 25 wk. While there was no significant difference between the WJCs or the BM-MSC group at time 0. b) Left ventricle to aorta outlet ratio (LA/Ao). In the WJC group, and not in the BM-MSC group, the LA/Ao ratio was significantly different from control animals. In all groups, the LA/Ao ratio significantly increased over time following MI. c) Left ventricle end diastolic diameter (LVd). Other variables measured including LVd, left ventricular systolic diameter, fractional shortening, etc were not significantly different between experimental groups (LVd is shown as an example). LVd, left ventricular end systolic diameter, and fractional shortening showed increases over time (as is shown for LVd here).

cytium formation, and co-culture of WJC with aBM-MSC did not. In support of our observations, Asumda and Chase showed that donor age impacts MSC plasticity and cardiogenic differentiation [45]. Similarly, in support of our observations, La Rocca et al. showed that WJCs may express the transcription factor GATA-4 [84]; GATA-4 is a critical transcription factor for cardiomyogenic differentiation [66]. Since we demonstrate that WJCs can undergo in vitro cardiomyogenic differentiation, our data support $\mathrm{Wu}$ et al.'s observation that human WJCs might assist recovery following MI by formation of cardiomyocytes in vivo [51]. We were able to pass differentiated cardiogenic cells twice and retain a few beating cells; however, following passage there was loss of the coordinated contracting ensemble (the passed cells could not re-form this ensemble). In the future, refinements to growth medium, substrate or other culture variables are needed to enhance the efficiency of cardiomyogenic differentiation, and enable re-establishment of higher-order organization between the contracting cells.

\section{Are WJCs Superior to BM-MSCs for Cellular Therapy?}

Several lines of evidence suggest that WJCs have certain advantages as a cell therapy for MI over aBM-MSC or fBMMSCs. First, in terms of generation of a clinical dose, in vi- 


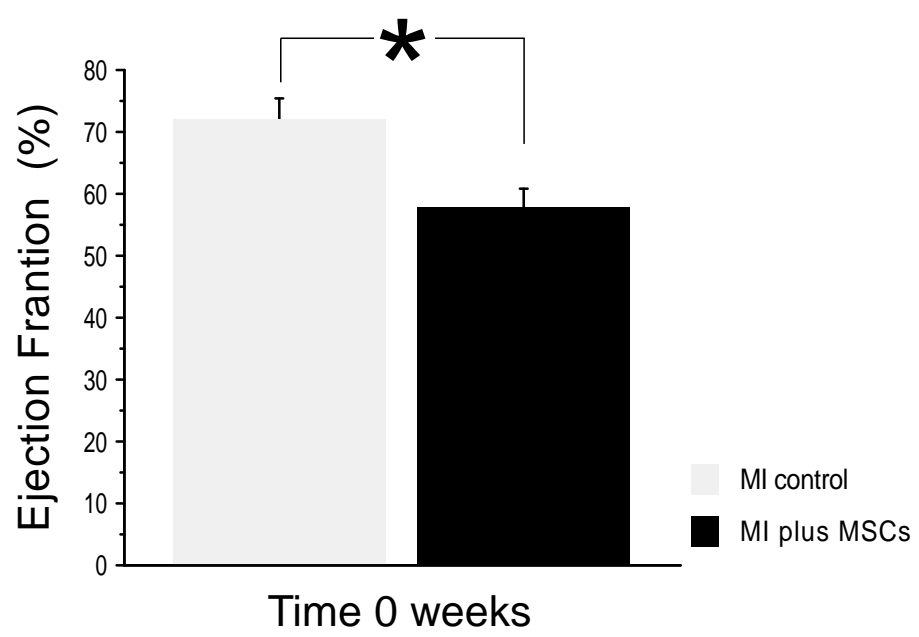

Fig. (6). Comparison of ejection fraction at time 0 between vehicle group and group that received mesenchymal stromal cells (MSCs). We assumed that randomized assignment to the experimental groups would result in ejection fraction being not significantly different between the vehicle group and group that received MSCs at time 0 . Note: that ejection fraction measured at time 0 was made before intravenous infusion of MSCs or vehicle. When compared to the vehicle group, the group that received MSCs (BM-MSCs and WJCs) had a significantly lower ejection fraction than the control at time $0(* \mathrm{p}<0.05)$.

tro expansion is a critical variable to consider. Previous work demonstrates that WJCs expand more rapidly and to a greater extent than aBM-MSCs prior to senescence [20,34]. Similarly, as the donor age increases, more difficulty is noted in establishing aBM-MSCs [62]. Here, we observed a similar pattern with rat fetal and adult MSCs. In a pilot study, we tried to establish BM-MSCs from 14 month old rats (old MSCs, data not shown). Compared to the aBMMSCs used here, old MSCs could not be established in culture and displayed a flattened, stellate morphology (YL and MLW, unpublished observation). In support of that observation, Asumda and Chase compared MSCs from 4 month and 14 month old rats and noted the flattened morphology and loss of tri-lineage differentiation potential in the older MSCs [45]. In addition to greater expansion potential, WJCs have greater colony forming unit- fibroblast (CFU-F) frequency than adult bone marrow-derived MSCs [23]. CFU-F is an indicator of self-renewal potential, a defining property of stem cells. In our investigation, co-culture of fBM-MSCs with WJCs produced rapid cardiomyogenic differentiation. In contrast, co-culture of aBM-MSC with WJCs did not produce cardiomyogenic differentiation. While others have reported that younger MSCs have cardiogenic differentiation potential superior to older MSCs [45, 65], our interpretation is that fBM-MSCs, through direct contact or via paracrine factors, can induce cardiomyogenic differentiation of WJCs. Together, these data suggest that WJCs have a broader differentiation potential than MSCs from old animals. For all these reasons, we suggest that WJCs may be a more advantageous adjunct for MI cell therapy.

In the present study, we compared aBM-MSCs and WJCs to test our hypothesis that WJCs would be superior for treatment following MI. A durable improvement in EF following MSC treatment was noted for both cell sources 25-32 weeks post MI. We found no conclusive difference between aBM-MSCs and WJCs as therapeutic cell sources. Based upon their impact on cardiac physiology, there is no clear advantage between the two MSC sources found in this preliminary study. However, WJCs have other features that need to be considered for their use as a therapy for treating MI. First, WJCs are collected safely, easily and painlessly at birth for later use as an allogeneic cell therapy. This is in contrast to fetal BM-MSCs or adult BM-MSCs, which require painful or invasive procedures to obtain. Fetal BMMSCs are rare and more controversial in their tissue source compared to discard umbilical cords, the source of WJCs. Second, WJCs can be readily expanded and cryopreserved to be ready to use an off-the-shelf allogeneic cellular therapy. Therefore, given that WJCs infused IV in the 24-48 hr window following MI home to sites of tissue damage and produce long-term positive effect upon cardiac function, we propose that IV WJCs may be a safe and effective off-theshelf cellular therapy for treating MI. We note that intracoronary human WJCs have been evaluated in humans with acute myocardial infarction (CLINICALTRIALS.GOV identifier: NCT01291329). To date, the study results have not been posted. Taken together, there are suggestions that WJCs are worthy of further investigation.

\section{Delivery of MSCs}

There are differences of opinion about therapeutic cell delivery for treating MI (for review see [85, 86]). Clearly, intracoronary or direct injection into the peri-infarction zone is an effective and efficient means to deliver cells to the heart [7], it is also the most invasive means of delivery. We demonstrated that WJCs home to damaged myocardial tissue following IV infusion: WJCs were found in the periinfarction zone following IV infusion and WJCs and aBMMSCs had a positive, durable functional effect 25-32 wks following MI. These results suggest that a therapeutic dose of MSCs has been delivered non-invasively. In support of this observation, others have shown that IV delivery of MSCs have positive effects following MI in rodent and 
swine models [49, 87]. Additionally, MSCs have therapeutic effects without significant homing to the damaged tissue via immune modulation actions such as via tumor necrosis factor $\alpha$ induced protein 6 (TSG-6), as is seen following corneal scarification [88] or following MI [89]. In terms of patient safety, IV delivery might improve the safety margin for cellular therapy. Further work is needed to confirm whether a dose-response relationship is found following IV MSC therapy and further work is needed determine whether IV TSG-6 works for treating $\mathrm{MI}$ in humans, mooting the point of direct or intracoronary injection. It may be necessary to demonstrate directly that the intracoronary delivery of TSG-6 or MSCs outweighs the safety and less invasive nature of IV delivery.

The timing of MSC delivery is likely to be a critical factor for efficacy of cell therapy. We delivered MSCs 24-48 hrs after MI. The non-invasive IV treatment lends itself to multiple doses if necessary and beneficial without incurring additional risk to an already damaged heart. Further work is necessary to experimentally support these notions.

\section{Assessing Heart Function Following Myocardial Infarc- tion}

We found that MSCs had a significant, positive effect on $\mathrm{EF}$ at $25 \mathrm{wks}$ and that positive effects lasted to $32 \mathrm{wks}$ (and perhaps as long as 68 wks after MI). In contrast, the LA/Ao ratio data showed a significant effect for WJCs, and no significant effect for BM-MSCs, compared to vehicle control. LA/Ao root diameter ratio is a measure of left atrial enlargement that has been associated to both heart failure and increased risk of death in humans and animals [90, 91]. While these data suggest a positive effect of WJC therapy upon some variables of cardiac function following MI, they do not clearly discriminate whether WJCs were more effective than aBM-MSCs. Similar to our observations, Wu et al. used echocardiography to assess the effect of human WJCs in the four weeks following MI [51]. Wu et al. found a significant improvement at both the two week and four week time point following MI compared to the control group. In contrast to the report by $\mathrm{Wu}$ et al., our MI rats demonstrated a decline in cardiac function (ejection fraction and other functional variables) over time (see Fig. 5). Our vehicle control rats showed progressive loss of cardiac function and left atrial enlargement. All the vehicle control rats died $\leq 32 \mathrm{wks}$ after MI. Our data suggest that MSC treatment might decrease or prevent the progressive loss of function following MI.

In this preliminary study, a small number of animals were assigned per group. Despite the small group size, we detected a significant impact of MSC therapy. Since rats initially compensate well following MI, and over time, more and more rats enter decompensated heart failure and die, this preliminary study should be followed with a larger study that evaluates in more depth the effect of MSCs on survival and provide a finer grain analysis of the components of cardiac function following MSC treatment.

In our study, post hoc analysis revealed that the animals that received MSCs might have had larger initial MIs than the vehicle control group (see Fig. 6), despite our randomiza- tion of group assignment. This observation suggests that MSCs might have had larger impact on heart function. Again, this suggests that this preliminary report should be followed on with study with larger group sizes to improve the statistical power and show more conclusively whether MSC treatment improved survival following MI and would clarify whether aBM-MSCs or WJCs are significantly different.

In conclusion, several lines of evidence presented suggest that IV MSCs might provide a positive, durable benefit when given in the 24-48 hour window following myocardial infarction. We found that the benefit of MSCs became significant $25 \mathrm{wks}$ after treatment and this was probably due to the progression nature of the MI injury in the control animals and the stemming of the progression in the MSC groups. We provided evidence that adult BM-MSCs and WJCs were effective on one measure of cardiac function (EF). We suggest that IV WJCs might be more advantageous than aBM-MSCs as an allogeneic, off-the-shelf therapy for MI. We found that co-culture of WJCs with fetal BM-MSCs induced rapid and robust differentiation along the cardiomyogenic lineage. The co-culture produced ensembles with coordinated contraction over the course of one week. Taken together, these data demonstrate that cardiac lineage development may be greatly improved by co-culturing MSCs from younger donors and suggest that MSCs from younger donors should be considered for regenerative therapy.

\section{CONFLICT OF INTEREST}

The authors confirm that this article content has no conflicts of interest.

\section{ACKNOWLEDGEMENTS}

YL was supported by the KSU graduate school travel stipend, the Jane Westfall Graduate Travel Award for Women, and received financial support from Universidad Centroccidental Lisandro Alvarado-Venezeula. The authors thank Dr. Marco Margiocco and Christy Zimmer for their assistance with echocardiography. The authors thank Josiah Cox for his support and assistance. This work was supported by grants from the Johnson Cancer Center, and NIH (R01 NS34160) to MLW and by support from the State of Kansas Legislature and the KINBRE. MLW acknowledges the support of BGW.

\section{SUPPLEMENTARY MATERIAL}

Supplementary material is available on the publishers Web site along with the published article.

\section{REFERENCES}

[1] Lopez AD, Mathers CD, Ezzati M, Jamison DT, Murray CJ. Global and regional burden of disease and risk factors, 2001: systematic analysis of population health data. Lancet 2006; 367(9524): 174757.

[2] Penn MS. Stem-cell therapy after acute myocardial infarction: the focus should be on those at risk. Lancet 2006; 367(9505): 87-8.

[3] Segers VF, Lee RT. Stem-cell therapy for cardiac disease. Nature 2008; 451(7181): 937-42.

[4] Tendera M, Wojakowski W. Clinical trials using autologous bone marrow and peripheral blood-derived progenitor cells in patients with acute myocardial infarction. Folia Histochem Cytobiol 2005; 43(4): $233-5$ 
[5] Assmus B, Schachinger V, Teupe C, et al. Transplantation of Progenitor Cells and Regeneration Enhancement in Acute Myocardial Infarction (TOPCARE-AMI). Circulation 2002; 106(24): 3009-17.

[6] Janssens S, Dubois C, Bogaert J, Theunissen K, Deroose C, Desmet $\mathrm{W}$, et al. Autologous bone marrow-derived stem-cell transfer in patients with ST-segment elevation myocardial infarction: double-blind, randomised controlled trial. Lancet 2006; 367(9505): 113-21.

[7] Kang S, Yang YJ, Li CJ, Gao RL. Effects of intracoronary autologous bone marrow cells on left ventricular function in acute myocardial infarction: a systematic review and meta-analysis for randomized controlled trials. Coron Artery Dis 2008; 19(5): 32735.

[8] Schachinger V, Erbs S, Elsasser A, et al. Intracoronary bone marrow-derived progenitor cells in acute myocardial infarction. $\mathrm{N}$ Engl J Med 2006; 355(12): 1210-21.

[9] Engelmann MG, Theiss HD, Hennig-Theiss C, et al. Autologous bone marrow stem cell mobilization induced by granulocyte colony-stimulating factor after subacute ST-segment elevation myocardial infarction undergoing late revascularization: final results from the G-CSF-STEMI (Granulocyte Colony-Stimulating Factor ST-Segment Elevation Myocardial Infarction) trial. J Am Coll Cardiol 2006; 48(8): 1712-21.

[10] Ince H, Nienaber CA. Granulocyte-colony-stimulating factor in acute myocardial infarction: future perspectives after FIRSTLINEAMI and REVIVAL-2. Nat Clin Pract Cardiovasc Med 2007; 4 Suppl 1: S114-S118.

[11] Lunde K, Solheim S, Aakhus S, et al. Intracoronary injection of mononuclear bone marrow cells in acute myocardial infarction. $\mathrm{N}$ Engl J Med 2006; 355(12): 1199-209.

[12] Ripa RS, Jorgensen E, Wang Y, et al. Stem cell mobilization induced by subcutaneous granulocyte-colony stimulating factor to improve cardiac regeneration after acute ST-elevation myocardial infarction: result of the double-blind, randomized, placebocontrolled stem cells in myocardial infarction (STEMMI) trial. Circulation 2006; 113(16): 1983-92.

[13] Zohlnhofer D, Ott I, Mehilli J, et al. Stem cell mobilization by granulocyte colony-stimulating factor in patients with acute myocardial infarction: a randomized controlled trial. JAMA 2006; 295(9): 1003-10.

[14] Povsic TJ, O'Connor CM. Cell therapy for heart failure: the need for a new therapeutic strategy. Expert Rev Cardiovasc Ther 2010; 8(8): 1107-26.

[15] Barlow S, Brooke G, Chatterjee K, et al. Comparison of human placenta- and bone marrow-derived multipotent mesenchymal stem cells. Stem Cells Dev 2008; 17(6): 1095-107.

[16] Battula VL, Bareiss PM, Treml S, et al. Human placenta and bone marrow derived MSC cultured in serum-free, b-FGF-containing medium express cell surface frizzled-9 and SSEA-4 and give rise to multilineage differentiation. Differentiation 2007; 75(4): 279-91.

[17] Li CD, Zhang WY, Li HL, et al. Isolation and Identification of a Multilineage Potential Mesenchymal Cell from Human Placenta. Placenta 2005 Sep 17. [Epub ahead of print].

[18] Montesinos JJ, Flores-Figueroa E, Castillo-Medina S, et al. Human mesenchymal stromal cells from adult and neonatal sources: comparative analysis of their morphology, immunophenotype, differentiation patterns and neural protein expression. Cytotherapy 2009; 11(2): 163-76.

[19] Pilz GA, Ulrich C, Ruh M, et al. Human term placenta-derived mesenchymal stromal cells are less prone to osteogenic differentiation than bone marrow-derived mesenchymal stromal cells. Stem Cells Dev 2011; 20(4): 635-46.

[20] Erickson GR, Gimble JM, Franklin DM, Rice HE, Awad H, Guilak F. Chondrogenic potential of adipose tissue-derived stromal cells in vitro and in vivo. Biochem Biophys Res Commun 2002; 290(2): 763-9.

[21] Gronthos S, Franklin DM, Leddy HA, Robey PG, Storms RW, Gimble JM. Surface protein characterization of human adipose tissue-derived stromal cells. J Cell Physiol 2001; 189(1): 54-63.

[22] Halvorsen YC, Wilkison WO, Gimble JM. Adipose-derived stromal cells--their utility and potential in bone formation. Int $\mathbf{J}$ Obes Relat Metab Disord 2000; 24 Suppl 4: S41-S44.
[23] Safford KM, Hicok KC, Safford SD, et al. Neurogenic differentiation of murine and human adipose-derived stromal cells. Biochem Biophys Res Commun 2002; 294(2): 371-9.

[24] Troyer DL, Weiss ML. Wharton's jelly-derived cells are a primitive stromal cell population. Stem Cells 2008; 26(3): 591-9.

[25] Baksh D, Yao R, Tuan RS. Comparison of proliferative and multilineage differentiation potential of human mesenchymal stem cells derived from umbilical cord and bone marrow. Stem Cells 2007; 25(6): 1384-92.

[26] Can A, Karahuseyinoglu S. Concise review: human umbilical cord stroma with regard to the source of fetus-derived stem cells. Stem Cells 2007; 25(11): 2886-95.

[27] Lu LL, Liu YJ, Yang SG, et al. Isolation and characterization of human umbilical cord mesenchymal stem cells with hematopoiesissupportive function and other potentials. Haematologica 2006; 91(8): 1017-26.

[28] Sarugaser R, Lickorish D, Baksh D, Hosseini MM, Davies JE. Human umbilical cord perivascular (HUCPV) cells: a source of mesenchymal progenitors. Stem Cells 2005; 23(2): 220-9.

[29] Weiss ML, Troyer DL. Stem cells in the umbilical cord. Stem Cell Rev 2006;2(2):155-62.

[30] Weiss ML, Anderson C, Medicetty S, et al. Immune properties of human umbilical cord Wharton's jelly-derived cells. Stem Cells 2008; 26(11): 2865-74.

[31] Angelucci S, Marchisio M, Di GF, et al. Proteome analysis of human Wharton's jelly cells during in vitro expansion. Proteome Sci 2010; 8: 18.

[32] Dominici M, Le BK, Mueller I, et al. Minimal criteria for defining multipotent mesenchymal stromal cells. The International Society for Cellular Therapy position statement. Cytotherapy 2006; 8(4): 315-7.

[33] Yang Z, Schmitt JF, Lee EH. Immunohistochemical analysis of human mesenchymal stem cells differentiating into chondrogenic, osteogenic, and adipogenic lineages. Methods Mol Biol 2011; 698: 353-66.

[34] Choong PF, Mok PL, Cheong SK, Leong CF, Then KY Generating neuron-like cells from BM-derived mesenchymal stromal cells in vitro. Cytotherapy 2007; 9(2): 170-83.

[35] Mitchell KE, Weiss ML, Mitchell BM, et al. Matrix cells from Wharton's jelly form neurons and glia. Stem Cells 2003; 21(1): 5060 .

[36] Anzalone R, Lo IM, Corrao S, et al. New emerging potentials for human Wharton's jelly mesenchymal stem cells: immunological features and hepatocyte-like differentiative capacity. Stem Cells Dev 2010; 19(4): 423-38.

[37] Anzalone R, Lo IM, Loria T, et al. Wharton's jelly mesenchymal stem cells as candidates for beta cells regeneration: extending the differentiative and immunomodulatory benefits of adult mesenchymal stem cells for the treatment of type 1 diabetes. Stem Cell Rev 2011; 7(2): 342-63.

[38] Asumda FZ, Chase PB. Nuclear cardiac troponin and tropomyosin are expressed early in cardiac differentiation of rat mesenchymal stem cells. Differentiation 2012; 83(3): 106-15.

[39] Guan J, Wang F, Li Z, et al. The stimulation of the cardiac differentiation of mesenchymal stem cells in tissue constructs that mimic myocardium structure and biomechanics. Biomaterials 2011; 32(24): 5568-80.

[40] Grabowska I, Brzoska E, Gawrysiak A, et al. Restricted myogenic potential of mesenchymal stromal cells isolated from umbilical cord. Cell Transplant 2012; 21(8): 1711-26.

[41] Lee JH, Kosinski PA, Kemp DM. Contribution of human bone marrow stem cells to individual skeletal myotubes followed by myogenic gene activation. Exp Cell Res 2005; 307(1): 174-82.

[42] Shi D, Reinecke H, Murry CE, Torok-Storb B. Myogenic fusion of human bone marrow stromal cells, but not hematopoietic cells. Blood 2004; 104(1): 290-4.

[43] Koninckx R, Hensen K, Daniels A, et al. Human bone marrow stem cells co-cultured with neonatal rat cardiomyocytes display limited cardiomyogenic plasticity. Cytotherapy 2009; 11(6): 77892.

[44] Martin-Rendon E, Sweeney D, Lu F, Girdlestone J, Navarrete C, Watt SM. 5-Azacytidine-treated human mesenchymal stem/progenitor cells derived from umbilical cord, cord blood and 
bone marrow do not generate cardiomyocytes in vitro at high frequencies. Vox Sang 2008; 95(2): 137-48.

[45] Asumda FZ, Chase PB. Age-related changes in rat bone-marrow mesenchymal stem cell plasticity. BMC Cell Biol 2011; 12: 44.

[46] Seshareddy K, Troyer D, Weiss ML. Method to isolate mesenchymal-like cells from Wharton's Jelly of umbilical cord. Methods Cell Biol 2008; 86: 101-19.

[47] Musch TI, Terrell JA. Skeletal muscle blood flow abnormalities in rats with a chronic myocardial infarction: rest and exercise. Am J Physiol 1992; 262(2 Pt 2): H411-H419.

[48] Dominici M, Le Blanc K, Mueller I, et al. Minimal criteria for defining multipotent mesenchymal stromal cells. The International Society for Cellular Therapy position statement. Cytotherapy 2006; 8(4): 315-7.

[49] Halkos ME, Zhao ZQ, Kerendi F, et al. Intravenous infusion of mesenchymal stem cells enhances regional perfusion and improves ventricular function in a porcine model of myocardial infarction. Basic Res Cardiol 2008; 103(6): 525-36.

[50] Ma J, Ge J, Zhang S, et al. Time course of myocardial stromal cellderived factor 1 expression and beneficial effects of intravenously administered bone marrow stem cells in rats with experimental myocardial infarction. Basic Res Cardiol 2005; 100(3): 217-23.

[51] Wu KH, Zhou B, Yu CT, et al. Therapeutic potential of human umbilical cord derived stem cells in a rat myocardial infarction model. Ann Thorac Surg 2007; 83(4): 1491-8.

[52] Li SC, Acevedo J, Wang L, et al. Mechanisms for progenitor cellmediated repair for ischemic heart injury. Curr Stem Cell Res Ther 2012; 7(1): 2-14.

[53] ter Horst KW. Stem cell therapy for myocardial infarction: are we missing time? Cardiology 2010; 117(1): 1-10.

[54] Wen Z, Zheng S, Zhou C, Wang J, Wang T. Repair mechanisms of bone marrow mesenchymal stem cells in myocardial infarction. $\mathrm{J}$ Cell Mol Med 2011; 15(5): 1032-43.

[55] Wollert KC, Drexler H. Cell therapy for the treatment of coronary heart disease: a critical appraisal. Nat Rev Cardiol 2010; 7(4): 20415.

[56] Le BK. Immunomodulatory effects of fetal and adult mesenchymal stem cells. Cytotherapy 2003; 5(6): 485-9.

[57] Nasef A, Ashammakhi N, Fouillard L. Immunomodulatory effect of mesenchymal stromal cells: possible mechanisms. Regen Med 2008; 3(4): 531-46.

[58] Uccelli A, Moretta L, Pistoia V. Immunoregulatory function of mesenchymal stem cells. Eur J Immunol 2006; 36(10): 2566-73.

[59] Pittenger MF, Martin BJ. Mesenchymal stem cells and their potential as cardiac therapeutics. Circ Res 2004; 95(1): 9-20.

[60] Caplan AI, Dennis JE. Mesenchymal stem cells as trophic mediators. J Cell Biochem 2006; 98(5): 1076-84.

[61] Caplan AI. Why are MSCs therapeutic? New data: new insight. J Pathol 2009; 217(2): 318-24.

[62] Clarke E, McCann SR. Age dependent in vitro stromal growth. Bone Marrow Transplant 1989; 4(5): 596-7.

[63] Paul D, Samuel SM, Maulik N. Mesenchymal stem cell: present challenges and prospective cellular cardiomyoplasty approaches for myocardial regeneration. Antioxid Redox Signal 2009; 11(8): 1841-55.

[64] Tang YL, Zhao Q, Zhang YC, et al. Autologous mesenchymal stem cell transplantation induce VEGF and neovascularization in ischemic myocardium. Regul Pept 2004; 117(1): 3-10.

[65] Zhang H, Fazel S, Tian H, et al. Increasing donor age adversely impacts beneficial effects of bone marrow but not smooth muscle myocardial cell therapy. Am J Physiol Heart Circ Physiol 2005; 289(5): H2089-H2096.

[66] Arminan A, Gandia C, Bartual M, et al. Cardiac differentiation is driven by NKX2.5 and GATA4 nuclear translocation in tissuespecific mesenchymal stem cells. Stem Cells Dev 2009; 18(6): 90718.

[67] Hakuno D, Fukuda K, Makino S, et al. Bone marrow-derived regenerated cardiomyocytes (CMG Cells) express functional adrenergic and muscarinic receptors. Circulation 2002; 105(3): 380-6.

[68] Huang Y, Zheng L, Gong X, et al. Effect of cyclic strain on cardiomyogenic differentiation of rat bone marrow derived mesenchymal stem cells. PLoS ONE 2012; 7(4): e34960.
[69] Li Z, Guo X, Palmer AF, Das H, Guan J. High-efficiency matrix modulus-induced cardiac differentiation of human mesenchymal stem cells inside a thermosensitive hydrogel. Acta Biomater 2012; 8(10): 3586-95.

[70] Makino S, Fukuda K, Miyoshi S, et al. Cardiomyocytes can be generated from marrow stromal cells in vitro. J Clin Invest 1999; 103(5): 697-705.

[71] Pereira WC, Khushnooma I, Madkaikar M, Ghosh K. Reproducible methodology for the isolation of mesenchymal stem cells from human umbilical cord and its potential for cardiomyocyte generation. J Tissue Eng Regen Med 2008; 2(7): 394-9.

[72] Medvinsky A, Smith A. Stem cells: Fusion brings down barriers. Nature 2003; 422(6934): 823-5.

[73] Zhang S, Wang D, Estrov Z, Raj S, Willerson JT, Yeh ET. Both cell fusion and transdifferentiation account for the transformation of human peripheral blood CD34-positive cells into cardiomyocytes in vivo. Circulation 2004; 110(25): 3803-7.

[74] Montesinos JJ, Flores-Figueroa E, Castillo-Medina S, et al. Human mesenchymal stromal cells from adult and neonatal sources: comparative analysis of their morphology, immunophenotype, differentiation patterns and neural protein expression. Cytotherapy 2009; 11(2): 163-76.

[75] Krampera M, Marconi S, Pasini A, et al. Induction of neural-like differentiation in human mesenchymal stem cells derived from bone marrow, fat, spleen and thymus. Bone 2007; 40(2): 382-90.

[76] Rosca AM, Burlacu A. Effect of 5-azacytidine: evidence for alteration of the multipotent ability of mesenchymal stem cells. Stem Cells Dev 2011; 20(7): 1213-21.

[77] Wang HS, Hung SC, Peng ST, et al. Mesenchymal stem cells in the Wharton's jelly of the human umbilical cord. Stem Cells 2004; 22(7): 1330-7.

[78] Degeorge BR, Jr., Rosenberg M, Eckstein V, et al. BMP-2 and FGF-2 synergistically facilitate adoption of a cardiac phenotype in somatic bone marrow c-kit+/Sca-1+ stem cells. Clin Transl Sci 2008; 1(2): 116-25.

[79] Li TS, Komota T, Ohshima M, et al. TGF-beta induces the differentiation of bone marrow stem cells into immature cardiomyocytes. Biochem Biophys Res Commun 2008; 366(4): 1074-80.

[80] Li Z, Gu TX, Zhang YH. Hepatocyte growth factor combined with insulin like growth factor-1 improves expression of GATA-4 in mesenchymal stem cells cocultured with cardiomyocytes. Chin Med J (Engl ) 2008; 121(4): 336-40.

[81] Muscari C, Bonafe F, Carboni M, et al. Difluoromethylornithine stimulates early cardiac commitment of mesenchymal stem cells in a model of mixed culture with cardiomyocytes. J Cell Biochem 2008; 103(4): 1046-52.

[82] Rota M, Kajstura J, Hosoda T, et al. Bone marrow cells adopt the cardiomyogenic fate in vivo. Proc Natl Acad Sci USA 2007; 104(45): 17783-8.

[83] Yoon J, Choi SC, Park CY, et al. Bone marrow-derived side population cells are capable of functional cardiomyogenic differentiation. Mol Cells 2008; 25(2): 216-23.

[84] La RG, Anzalone R, Corrao S, et al. Isolation and characterization of Oct-4+/HLA-G+ mesenchymal stem cells from human umbilical cord matrix: differentiation potential and detection of new markers. Histochem Cell Biol 2009; 131(2): 267-82.

[85] Trivedi P, Tray N, Nguyen T, Nigam N, Gallicano GI. Mesenchymal stem cell therapy for treatment of cardiovascular disease: helping people sooner or later. Stem Cells Dev 2010; 19(7): 1109-20.

[86] Williams AR, Hare JM. Mesenchymal stem cells: biology, pathophysiology, translational findings, and therapeutic implications for cardiac disease. Circ Res 2011; 109(8): 923-40.

[87] Cheng Z, Ou L, Zhou X, et al. Targeted migration of mesenchymal stem cells modified with CXCR4 gene to infarcted myocardium improves cardiac performance. Mol Ther 2008; 16(3): 571-9.

[88] Roddy GW, Oh JY, Lee RH, et al. Action at a distance: systemically administered adult stem/progenitor cells (MSCs) reduce inflammatory damage to the cornea without engraftment and primarily by secretion of TNF-alpha stimulated gene/protein 6 . Stem Cells 2011; 29(10): 1572-9. 
[89] Lee RH, Pulin AA, Seo MJ, et al. Intravenous hMSCs improve myocardial infarction in mice because cells embolized in lung are activated to secrete the anti-inflammatory protein TSG-6. Cell Stem Cell 2009; 5(1): 54-63.

[90] Borgarelli M, Savarino P, Crosara S, et al. Survival characteristics and prognostic variables of $\operatorname{dogs}$ with mitral regurgitation attributable to myxomatous valve disease. J Vet Intern Med 2008; 22(1): $120-8$

[91] Tanabe K, Yamaguchi K, Tani T, et al. Left atrial volume: predictor of atrial fibrillation in patients with degenerative mitral regurgitation. J Heart Valve Dis 2007; 16(1): 8-12. 